\title{
Modelle der Oberflächenform mechanisch bearbeiteter Werkstücke für die Messunsicherheitsbestimmung durch Simulation bei taktilen Koordinatenmessungen
}

\author{
Thomas Kistner ${ }^{1}$, Dr. Dietrich Imkamp ${ }^{1}$, Prof. Tino Hausotte ${ }^{2}$ \\ ${ }^{1}$ Carl Zeiss IMT GmbH, Carl-Zeiss-Straße 22, 73447 Oberkochen, Deutschland \\ ${ }^{2}$ Lehrstuhl für Fertigungsmesstechnik, Friedrich-Alexander-Universität Erlangen-Nürnberg (FAU), \\ Nägelsbachstraße 25, 91052 Erlangen
}

\section{Zusammenfassung}

Die Messunsicherheitsermittlung durch Simulation - bekannt unter dem Begriff „virtuelles Koordinatenmessgerät" - ist eine hoch entwickelte Methode im Bereich der Koordinatenmesstechnik. Durch Weiterentwicklung sind neue Funktionen zur Abschätzung von bisher nicht betrachteten Einflussgrößen hinzugekommen. Damit kann unter anderen der Einfluss der Messstrategie auf das Messergebnis in Abhängigkeit von der Formabweichung der betrachteten Werkstückoberfläche abgeschätzt werden. Durch Modellierung auf Basis von Informationen über die Bearbeitung werden die Werkstückoberflächen digitalisiert und können im Rahmen der Messunsicherheitsermittlung mit dem virtuellen Koordinatenmessgerät verwendet werden.

Keywords: Fertigungsmesstechnik, Messunsicherheit, Monte-Carlo-Simulation, Formmodellierung

\section{Einleitung}

Die Koordinatenmesstechnik dient zur dimensionellen Messung von Werkstücken vorwiegend im Rahmen der Qualitätsprüfung. Mit dem Begriff Koordinatenmesstechnik werden alle Messverfahren bezeichnet, welche das zu messende Objekt durch Koordinatenpunkte im Raum erfassen. Klassische Koordinatenmessgeräte sind kartesisch aufgebaut, daher beziehen sich die erfassten Koordinaten vorwiegend auf ein kartesisches Koordinatensystem. Bei taktilen Messungen werden die Punkte mit einem meist kugelförmigen Antastelement erfasst. Das Antastelement berührt das Werkstück und erkennt beim Erreichen einer vordefinierten Messkraft den Kontaktpunkt zwischen Werkstück und Antastelement als Messpunkt. Im Einzelpunktmodus werden die Messpunkte durch eine Antastung an einer definierten Stelle erfasst. Im Anschluss an die Punkerfassung löst das Tastelement den Kontakt mit der Werkstückoberfläche. Im Scanningmodus bleibt das Antastelement in Kontakt mit dem Werkstück, verfährt entlang einer definierten Messbahn und nimmt dabei kontinuierlich Messpunkte auf. Aus den erfassten Messpunkten wird durch Ausgleichsrechnung das assoziierte Element und damit das Ergebnis einer Messung berechnet. Dieses Ergebnis dient als Grundlage für die Bewertung der Qualität des gemessenen Objekts. Dabei wird in einem der Messwert mit dem Sollwert verglichen.

Der Messwert setzt sich aus dem wahren Wert sowie zufälligen und systematischen Messabweichungen zusammen. Zufällige Abweichungen sind im Gegensatz zu den systematischen Abweichungen nicht korrigierbar. Der Einfluss systematischer Komponenten auf das Messergebnis kann dagegen ausgeglichen werden. Dafür sind Kenntnis über den Messvorgang und seine Einflussparameter erforderlich. Aus diesen Informationen wird die Unsicherheit berechnet. Der Messwert wird damit um seine Unsicherheit erweitert.

In Forschung und Industrie spielt die Kenntnis der Messunsicherheit und ihre Größenordnung eine entscheidende Rolle Die Kenntnis über die eigenen Prozesse und deren Fähigkeit wird in Qualitätsrichtlinien gefordert. Die Messunsicherheit ist hierbei ein Indikator für deren Beurteilung. Die Kenntnis der Messunsicherheit kann neben dem Nachweis zur Eignung auch zur Verbesserung von Messprozessen genutzt werden.

\section{Messunsicherheitsbestimmung mit dem virtuellen Koordinatenmessgerät}

Die Bestimmung der Messunsicherheit durch Simulation basiert auf einer umfassenden Modellierung der Messaufgabe. Die Simulation löst das Problem der komplexen Überlagerung vieler Einflussgrößen unter Zuhilfenahme der 
Wahrscheinlichkeitstheorie [2]

Mathematischer Hintergrund ist dabei die Monte-Carlo-Simulation. Die Umsetzung dieser Methodik für Koordinatenmessungen ist durch das virtuelle Koordinatenmessgerät, abgekürzt "VCMM" (Virtual Coordinate Measuring Machine), realisiert [3]. Das VCMM befindet sich seit 2004 in Forschung und Industrie im Einsatz. Anwendung findet es hauptsächlich in Kalibrierlaboratorien. Durch die 2018 fertiggestellte Weiterentwicklung des virtuellen Koordinatenmessgeräts wurde dessen Funktionsumfang durch die Berücksichtigung von Scanning-Messungen und Tasterwechsel erweitert [4]. Die Simulation ist heute so in die Messsoftware der Hersteller integriert, dass bestehende Messprogramme im Anschluss an die Messung oder zu einem beliebigen späteren Zeitpunkt mit dem VCMM ausgewertet werden können. Die berechnete Messunsicherheit wird begleitend zum Messwert im Protokoll der Messung ausgegeben.

Die gerätebezogenen Einflussgrößen (z. B.: Restabweichungen der Führungen) werden bei der Installation des VCMM ermittelt und bei Änderungen am Gerät aktualisiert. Messaufgabenbezogene Größen (z. B.: Werkstücktemperatur) werden durch den Anwender direkt vor der Durchführung der Messaufgabe bestimmt. $\mathrm{Zu}$ den messaufgabenbezogenen Größen gehört auch die Oberflächenrauheit, die letztlich nur durch eine sehr hohe Anzahl von Messpunkten vollständig erfassbar ist. Der Abstand der Messpunkte ist so klein zu wählen, dass der Übergang von der Rauheit zu den Anteilen mit noch kürzeren Wellenlängen erfasst wird. Mit dieser Messstrategie wird eine ausreichende Strukturauflösung $\quad(<\lambda s$ unter der Grenzwellenlänge nach [5]) erzielt.

Um für den praktischen Einsatz die Anzahl der Messpunkte zu begrenzen, und die Reproduzierbarkeit der Messung zu steigern, wird durch die punktgenaue Festlegung der Messstrategie als Teil der Messaufgabendefinition der Einfluss der Oberflächenform inklusive der Oberflächenwelligkeit (gesamtes Spektrum der Oberflächenformabweichungen gemäß Bild 8 in [6]) eliminiert. Dieses Vorgehen ist beim Kalibrieren von Normalen zweckmäßig und etabliert. Bei der Messung von Werkstücken, bei denen die unvollständige Erfassung der Oberfläche durch eine begrenzte Messpunktanzahl („Unterabtastung“) zur Messunsicherheit beiträgt, ist das allerdings nicht zielführend.

\section{Beitrag der Messstrategie}

Eine Werkstückoberfläche ist nur dann vollständig gemessen, wenn jede Oberflächencharakteristik bei der taktilen Messung erfasst wird. Bei einer unvollständigen Erfassung kann es vorkommen, dass Oberflächencharakteristika nicht erfasst werden und das Messergebnis somit unvollständig ist. Handelt es sich dabei um dominante Charakteristika, kann das Messergebnis dadurch verfälscht werden. Abweichungen hierbei betreffen sowohl Maß, Form und Lage des gemessenen Elements.

Durch die Analyse des Einflusses der Unterabtastung wird der Beitrag der Messstrategie (Messpunktverteilung) zur Unsicherheit abgeschätzt und im VCMM berücksichtigt [7]. Dazu werden Messungen mit verschiedenen Messstrategien miteinander verglichen. Das VCMM generiert für jedes Messmerkmal ein eigenes Formmodell. Dieses Formmodell wird entsprechend der zu erwarteten Formabweichung als sinusförmige Oberfläche modelliert und in Amplitude und Ortswellenlänge skaliert. Die Messmerkmale werden mit der vom Anwender als Standard für die Messung definierten Punktverteilung virtuell abgetastet.

Nach dieser virtuellen Messung findet die Messunsicherheitsermittlung durch das VCMM statt. Hierbei werden alle relevanten Einflüsse auf die Messung berücksichtigt. Im Anschluss an diese Standardmessung findet eine Simulation der Messung mit einer vielfachen Anzahl an Messpunkten statt. Diese Anzahl sollte so gewählt werden, dass die kürzeste Ortswellenlänge der Oberflächenform mit mindestens sieben Punkten [6] erfasst wird. Diese simulierte Messung dient zur vollständigen Erfassung der Formabweichung und wird als Referenz betrachtet. Der Unterschied der erfassten Formabweichungen bei den Messungen stellt den zusätzlichen Beitrag durch die Unterabtastung dar und fließt in die Messunsicherheit mit ein.

In Abbildung 1 werden solche simulierten Messungen mit den jeweils erfassten Formabweichungen dargestellt. Als Modell wird eine geschliffene Werkstückoberfläche (Werkzeug mit geometrisch unbestimmter Schneide) verwendet. Das erste (oberste) Bild der Abbildung zeigt die tatsächliche Oberfläche und die Berührpunkte mit $2 \mathrm{~mm}$ TasterDurchmesser. Die Oberfläche wird mit insgesamt 20000 Punkten simuliert und weist eine Formabweichung von insgesamt 7,4 $\mu \mathrm{m}$ auf. Im zweiten und dritten Bild der Abbildung wird das bisher im virtuellen Koordinatenmessgerät implementierte 

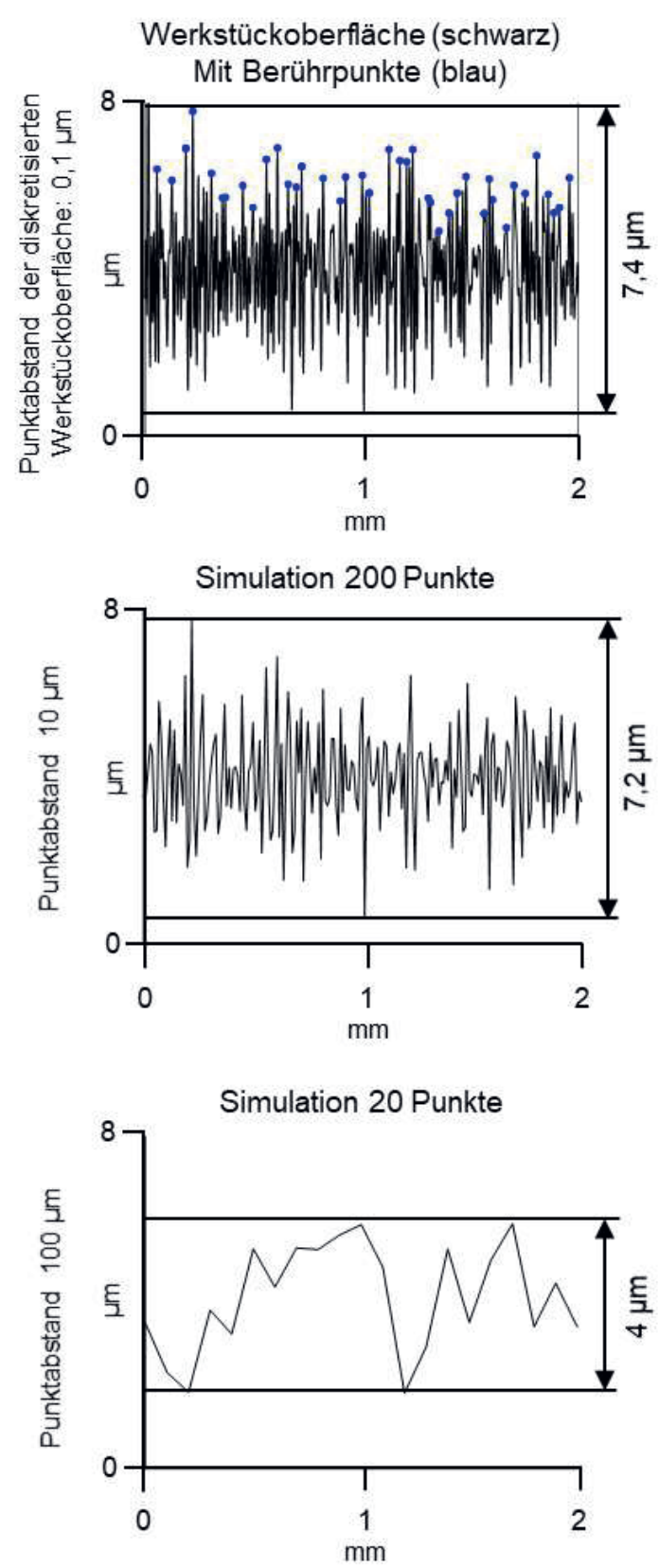

Simulierte Messung mit Tastkugeldurchmesser $2 \mathrm{~mm}$

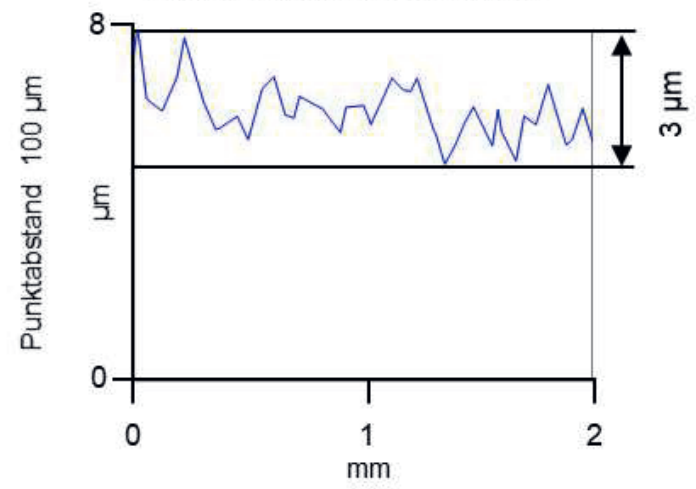

Abb. 1: Wirkung der Unterabtastung: Vergleich verschiedener Punktedichten und Herangehensweisen
Vorgehen gezeigt. Die Abtastung durch die Referenzmessung erfolgt mit einer vielfachen Punkteanzahl im Vergleich zur Standardmessung. Die sich ergebende Differenz der Formabweichung stellt den Beitrag durch Unterabtastung dar.

Im zweiten Bild der Abbildung werden Punkte für die Erfassung der Referenz mit dem Punktabstand von $10 \mu \mathrm{m}$ aus der originalen Werkstückoberfläche extrahiert. Hierbei wird eine Formabweichung von 7,2 $\mu \mathrm{m}$ erfasst. Im dritten Bild der Abbildung werden die Punkte mit einem Punktabstand von $100 \mu \mathrm{m}$ aus der originalen Werkstückoberfläche extrahiert. Dies stellt den vom Anwender festgelegten Messpunktabstand der beispielhaften Messaufgabe dar. Die erfasste Formabweichung dieser Standardmessung ist mit $4 \mu \mathrm{m}$ deutlich geringer als die der Referenzmessung. Man erkennt, dass die Oberflächencharakteristik nicht mehr vollständig abgebildet wird. Der Beitrag durch Unterabtastung ergibt sich aus der Differenz der erfassten Formabweichungen von Standard und Referenz. Er beträgt hier 3,2 $\mu \mathrm{m}$.

Im vierten Bild der Abbildung werden die Punkte nicht einfach aus der originalen Werkstückoberfläche extrahiert, sondern die mechanische Filterung des kreisförmigen Antastelements mit dem Durchmesser $2 \mathrm{~mm}$ wird mit einberechnet. Das erfasste Profil dieser simulierten Messung ergibt sich aus den Berührpunkten des Tastelements mit der Werkstückoberfläche. Durch die Geometrie des Antastelements werden die Täler der Werkstückoberfläche nicht mehr erfasst. Die erfasste Formabweichung beträgt nur noch $3 \mu \mathrm{m}$. Durch die Erfassung der Messpunkte an den Profilspitzen ist ein zudem ein Lageversatz der Messpunkte im Vergleich zum bisher implementierten Vorgehen erkennbar.

Weitere Untersuchungen werden zukünftig für Fertigungsverfahren mit geometrisch bestimmter und geometrisch unbestimmter Schneide durchgeführt. Bei den simulierten Messungen wird die Werkstückoberfläche durch das kreisförmig modellierte Tastelement mechanisch gefiltert. Bei kleinen Tastelementen findet eine detailliertere Erfassung der Werkstückoberfläche statt. Bei großen Tastelementen werden meist nur noch die Spitzen des Profils erfasst. Die erfassbare Formabweichung verringert sich somit mit der Vergrößerung des Tastelements. Die Unterschätzung der Form wird in der Gegenüberstellung der verschiedenen Messstrategien deutlich sichtbar. Findet eine ausreichende Erfassung der Werkstückoberfläche durch die Messstrategie 
des Anwenders statt, sind die Unterschiede zwischen Referenz- und Standardmessstrategie zu vernachlässigen.

\section{Formmodellierung}

Durch die individuelle Anpassung des Formmodells an die Parameter des Fertigungsprozesses können aussagekräftige Angaben für diesen Beitrag zur Messunsicherheit gemacht werden. Bei der Modellierung wird zwischen Fertigungsverfahren mit geometrisch bestimmter und geometrisch unbestimmter Schneide unterschieden.

Erstere, zum Beispiel Fräsen und Drehen, werden anhand der Fertigungsparameter modelliert. Hierfür werden weitergehende Kenntnisse über die Eigenschaften der resultierenden Werkstückoberfläche aus den jeweiligen Fertigungsprozessen (z. B.: Vorschub und Schneidkantenradius [8]) genutzt. Diese Fertigungsverfahren sind im Hinblick auf die daraus entstehenden Oberflächenstrukturen bei einem idealen Prozess ausreichend bekannt.

Fertigungsverfahren mit geometrisch unbestimmter Schneide erzeugen eine zufällige Werkstückoberfläche. Beschreibungen der resultierenden Werkstückoberfläche bei perfekten Prozessparametern sind durch die Zufälligkeit des Fertigungsprozesses, resultierend aus der jeweiligen Schneidengeometrie der strukturgebenden Werkzeuge, nicht bekannt. Werkstückoberflächen mit diesen Eigenschaften werden daher durch ein Modell basierend auf sich überlagernden Sinuswellen erstellt. Die Informationen über die Parameter Amplitude, und Wellenlänge kommen aus Analysemessungen realer Bauteile mit Tastschnittgeräten mit einem Punktabstand $<0,1 \mu \mathrm{m}$. Gemäß dem Zufallscharakter der Oberfläche wird daraus ein Modell nicht statisch erstellt, sondern mit jedem Simulationsdurchlauf variiert. Ein einzelnes Modell besteht aus 82 verschiedenen Wellenlängen. Jeder Wellenlänge sind eine Amplitude mit oberen und unterm Grenzwert und eine zufällig generierte Phasenlage zugeordnet. Beide Modellierungsarten dienen der Simulation der aus dem Fertigungsprozess entstehenden Oberflächencharakteristik. Der Punktabstand der initial simulierten Werkstückoberflächen beträgt $0,1 \mu \mathrm{m}$. Dieser Abstand entspricht den Anforderungen an den Profilpunktabstand bei der Messung mit einem Tastschnittgerät [9]. Zudem ist der Abstand ausreichend gering, um die Oberfläche in Hinblick auf taktile Koordinatenmessungen mit kugelförmigen

Antastelementen (Tastkugeldurchmesser $>0,2 \mathrm{~mm}$ ) vollständig $\mathrm{zu}$ erfassen. Langwellige Abweichungen, welche z. B. aus Führungsabweichungen der Werkzeugmaschine stammen, werden in der Modellierung nicht berücksichtigt. Im Anschluss an die jeweilige Modellierung wird die mechanische Abtastung durch ein kugelförmiges Tastelement mittels morphologischer Filterung mit einem Kreis simuliert.

\section{Verifikation des Modells}

Die Verifikation der Modelle und der Vorgehensweise erfolgt durch den Vergleich mit Messungen an Oberflächennormalen (Oberflächenvergleichsmuster [10]). Neben Dreh- und Fräsprofilen als Vertreter der Fertigungsverfahren mit geometrisch bestimmter Schneide werden geschliffene Profile, Fertigungsverfahren mit geometrisch unbestimmter Schneide, betrachtet. Die Fertigungsparameter dieser Normale sind bekannt. Die Messung der Normale erfolgt mit verschiedengroßen, kugelförmigen Antastelementen, um die Bandbreite möglicher Messaufgaben, die mit unterschiedlichen Antastelementen ausgeführt werden, abzudecken. Die Messstrecke beträgt je nach Profil bis zu $30 \mathrm{~mm}$. Die folgende Darstellung beschränkt sich auf den Schleifprozess.

Abbildung 2 zeigt ein Beispiel für den Vergleich von simulierten Messungen an einem Oberflächenmodell eines Schleifprozesses mit Messungen an einer realen Oberfläche, die ähnliche Fertigungsparameter wie das Modell aufweist. Die Profile werden dafür jeweils in einem Ausschnitt von $2 \mathrm{~mm}$ Länge dargestellt. Der Punktabstand der in Abbildung 3 gezeigten Beispiele beträgt jeweils $10 \mu \mathrm{m}$. Das gemessene Oberflächenprofil wurde mit einem Gauß-Hochpassfilter gefiltert, um langwellige Formabweichungen aus dem Fertigungsprozess zu eliminieren, und die Vergleichbarkeit zwischen beiden Profilen herzustellen. Die Messung wurde mit einem Antastelement mit dem Durchmesser $2 \mathrm{~mm}$ simuliert und an einem Koordinatenmessgerät durchgeführt. Aufgrund langwelliger Verformungen des gemessenen Oberflächennormals wurde das Profil mit einem Hochpass-Gaußfilter mit der Grenzwellenlänge von 2,5 mm gefiltert. 
Simuliertes Profil (schwarz) + Berührpunkte Taster $\varnothing 2 \mathrm{~mm}$ (blau)

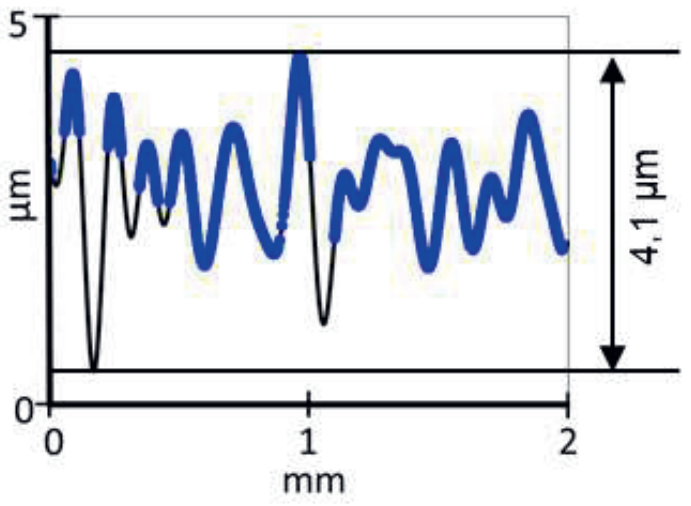

Simulierte Messung mit Taster $\varnothing 2 \mathrm{~mm}$

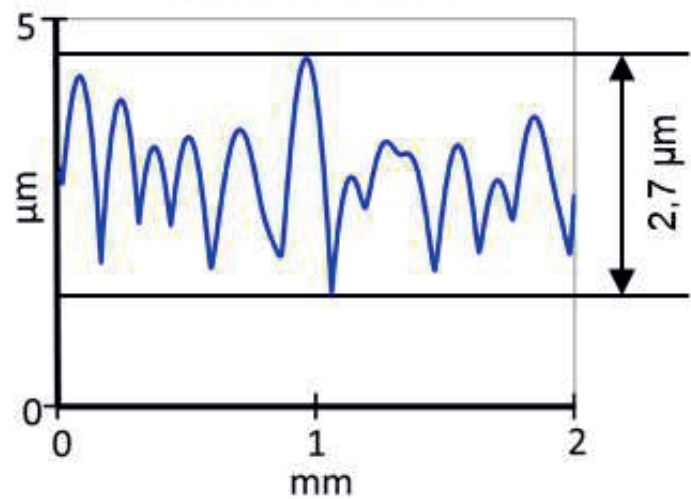

Reale Messung mit Taster Ø $2 \mathrm{~mm}$

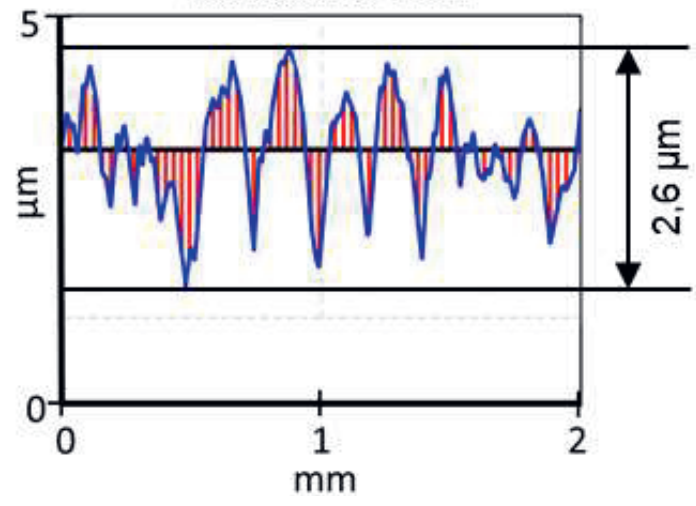

Abb. 2: Auszug einer beispielhaften Modellierung eines Schleifprofils: Simuliertes Ausgangs- und Messprofil im Vergleich zu einer real gemessener geschliffenen Werkstückoberfläche unter Berücksichtigung des mechanischen Filters durch das Tastelement (Durchmesser $2 \mathrm{~mm}$ )

Ein in Hinblick auf die mechanische Filterung relevanter Vergleichsparameter ist die mittlere Rillenbreite der Profilelemente $(R s m)$ und die mittlere Höhe der Profilelemente $(R c)$. Durch diese Parameter wird die Eintauchtiefe der Tastkugel in das gemessene Profil maßgeblich bestimmt. Die mittlere Rillenbreite Rsm beträgt $0,220 \mathrm{~mm}$ bei dem simulierten Schleifprofil und $0,226 \mathrm{~mm}$ bei dem real gemessenen Schleifprofil. Die mittlere Höhe der Profilelemente $R c$ beträgt $1,78 \mu \mathrm{m}$ bei dem simulierten Schleifprofil und 1,791 $\mu \mathrm{m}$ bei dem real gemessenen Schleifprofil. Die Abweichungen zwischen den Profilen sind kleiner $3 \%$ für $R s m$ und kleiner $1 \%$ für Rc. Die Auswertungen beziehen sich jeweils auf die vollständigen Profile und nicht nur auf den in Abbildung 3 beispielhaft dargestellten Auszug.

Das gemessene Profil zeigt ein hochfrequentes Rauschen. Dieses Rauschen entsteht bei der realen Messung und wird bei der Simulation der Messung nicht erzeugt. Zukünftig ist es denkbar, auf die simulierten Modelle zusätzliche kurzwellige Störfrequenzen aufzubringen, um eine noch höhere Vergleichbarkeit mit taktilen Koordinatenmessungen herzustellen. Die Verifikation der in Simulation und Realität gemessenen Profile findet über die Parameter Rsm und Rc statt. Weitere Oberflächenparameter zur Verifikation sind Gegenstand der derzeitigen Untersuchung und können zukünftig mit hinzugezogen werden.

\section{Ausblick}

Durch die Modellierung der Werkstückoberfläche, die Simulation unterschiedlicher Messpunktverteilungen sowie der mechanischen Filterwirkung und der sich anschließenden Bewertung der Messstrategie auf Basis der Form der Profile können messaufgabenspezifisch Messstrategien hinsichtlich der Messunsicherheit in Verbindung mit dem VCMM optimiert werden. Weiterhin können die Modelle der Fertigungsverfahren zukünftig in das virtuelle Koordinatenmessgerät integriert werden. Dies ermöglicht dem Anwender die Abschätzung des Beitrags der Unterabtastung zur Messunsicherheit unter Verwendung realer Oberflächenformmodelle an seinen konkreten Messaufgaben. Die Integration der Messstrategie als Parameter zur Messunsicherheitsbetrachtung wird damit unterstützt. 


\section{Literaturnachweis}

[1] Weckenmann, A. (Hrsg): Koordinatenmesstechnik: Flexible Messstrategien für Maß, Form und Lage; 2. Aufl. München : Hanser Verlag, 2012.

[2] JGCM 101:2008: Evaluation of measurement data - Supplement 1 to the "Guide to the expression of uncertainty in measurement" Propagation of distributions using a Monte Carlo method, JGCM (Joint Committee for Guides in Metrology), 2008. (Internet: http://www.bipm.org/en/publications/ guides/gum.html).

[3] Kistner, T.; Gabbia, A.; Imkamp, D: Messunsicherheit durch Simulation: Stand der Technik und neue Entwicklungen für die Koordinatenmesstechnik in der industriellen Produktion, 18. GMA/ITG-Fachtagung Sensoren und Messsysteme 2016, Nürnberg, 2016.

[4] Franke, M.; Kistner, T.; Hausotte, T.; Heißelmann, D.; Schwehn, C.; Wendt, K.: Bestimmung der Messunsicherheit für Koordinatenmesssysteme. tm - Technisches Messen, 84(5), S. 325-335.

[5] DIN EN ISO 4287:2010-07-00 Geometrische Produktspezifikationen (GPS) Oberflächenbeschaffenheit: Tastschnittverfahren - Benennungen, Definitionen und Kenngrößen der Oberflächenbeschaffenheit (ISO 4287:1997 + Cor 1:1998 + Cor 2:2005 + Amd 1:2009); Deutsche Fassung EN ISO 4287:1998 + AC;2008 + A1:2009.

[6] VDI/VDE-Richtlinie 2631 Blatt 3, Formprüfung, Eigenschaften und Auswahl von Filtern, September 2018.

[7] Heißelmann, D.; Franke, M.; Kistner, T.; Schwehn, C.: Das virtuelle Koordinatenmessgerät VCMM, Koordinatenmesstechnik 2018 - Technologien und Anwendung, DIN-Tagung, Braunschweig, 2018.

[8] Klocke, F.; König, W.: Fertigungsverfahren 1: Drehen, Fräsen, Bohren, 4. Aufl. BerlinHeidelberg : Springer, 2007. - ISBN 978-3-54023458-6.

[9] DIN EN ISO 3274:1997 Geometrische Produktspezifikationen (GPS) Oberflächenbeschaffenheit: Tastschnittverfahren - Nenneigenschaften von Tastschnittgeräten (ISO 3274:1996); Deutsche Fassung EN ISO 3274:1997.

[10] ISO 2632-1:1985 Roughness comparison specimens - Part 1: Turned, Ground, Bored, Milled, Shaped and Planed, September 1985.
[11] DIN EN ISO 3274:1997 Geometrische Produktspezifikationen (GPS) Oberflächenbeschaffenheit: Tastschnittverfahren - Oberflächen mit plateauartigen funktionsrelevanten Eigenschaften - Teil 2: Beschreibung der Höhe mittels linearer Darstellung der

Materialanteilkurve (ISO 1356-2:1996);

Deutsche Fassung EN ISO 13565-2:1997. 